\title{
OPEN Time-of-day-dependent responses of cyanobacterial cellular viability against oxidative stress
}

\author{
Kenya Tanaka ${ }^{1}{ }^{1}$, Ginga Shimakawa ${ }^{2,3}$ \& Shuji Nakanishi ${ }^{1,3 凶}$
}

As an adaptation to periodic fluctuations of environmental light, photosynthetic organisms have evolved a circadian clock. Control by the circadian clock of many cellular physiological functions, including antioxidant enzymes, metabolism and the cell cycle, has attracted attention in the context of oxidative stress tolerance. However, since each physiological function works in an integrated manner to deal with oxidative stress, whether or not cell responses to oxidative stress are under circadian control remains an open question. In fact, circadian rhythms of oxidative stress tolerance have not yet been experimentally demonstrated. In the present work, we applied an assay using methyl viologen (MV), which generates reactive oxygen species (ROS) under light irradiation, and experimentally verified the circadian rhythms of oxidative stress tolerance in photosynthetic cells of the cyanobacterium Synechococcus elongatus PCC 7942, a standard model species for investigation of the circadian clock. Here, we report that ROS generated by MV treatment causes damage to stroma components and not to the photosynthetic electron transportation chain, leading to reduced cell viability. The degree of decrease in cell viability was dependent on the subjective time at which oxidative stress was applied. Thus, oxidative stress tolerance was shown to exhibit circadian rhythms. In addition, the rhythmic pattern of oxidative stress tolerance disappeared in mutant cells lacking the essential clock genes. Notably, ROS levels changed periodically, independent of the MV treatment. Thus, we demonstrate for the first time that in cyanobacterial cells, oxidative stress tolerance shows circadian oscillation.

Many oxygenic photosynthetic organisms have evolved circadian clocks to adapt to the predictable, $24 \mathrm{~h}$ changes in light levels. Since daily fluctuations in environmental light levels lead to periodic variations in reactive oxygen species (ROS) levels in photosynthetic organisms, the physiological function of the circadian clock has been thought to be relevant to oxidative stress tolerance. For example, the activity or redox state of antioxidant enzymes are regulated by the circadian clock in plant ${ }^{1,2}$. Moreover, it has been suggested that circadian gating of cell division at a suitable time protects cells from photosynthetic oxidative stress in unicellular red alga ${ }^{3}$.

As mentioned above, the relationship between the circadian clock and oxidative stress has been described in studies of individual functions. However, since each adaptive function works in concert in natural environments, the effects of the circadian clock on cellular oxidative stress tolerance cannot be determined by analyzing each function. Although circadian control is important for cell physiology, the impact of circadian control of physiological systems on cells has not yet been fully elucidated.

The cyanobacterium Synechococcus elongatus PCC 7942 (hereafter, S. elongatus), a photosynthetic autotrophic bacterium, is an appropriate model for study of circadian control of cellular physiology because the molecular mechanisms underlying its circadian clock is well understood, as summarized in recent reviews ${ }^{4,5}$. In this organisms, attention has been also paid to relationship between circadian clock and oxidative stress. For example, it has been reported that circadian regulation of energy metabolism keeps ROS levels low ${ }^{6}$. Although the circadian clock is known to regulate the expression patterns of many genes, this regulation is not necessarily reflected in protein abundance $\mathrm{e}^{7,8}$. In addition, ultradian metabolic rhythm was observed in Cyanothece sp. ATCC 51142, indicates that circadian clock does not always produce a 24 -h rhythmic phenotype ${ }^{9}$. Moreover, to date, no phenotype showing circadian rhythm in oxidative stress tolerance has been experimentally confirmed.

\footnotetext{
${ }^{1}$ Graduate School of Engineering Science, Osaka University, 1-3 Machikaneyama, Toyonaka, Osaka 560-8631, Japan. ${ }^{2}$ Institute for Integrative Biology of the Cell (I2BC), CEA, CNRS, Université Paris-Sud, Université Paris-Saclay, 91198 Gif-sur-Yvette cedex, France. ${ }^{3}$ Research Center for Solar Energy Chemistry, Osaka University, 1-3 Machikaneyama, Toyonaka, Osaka 560-8631, Japan. ${ }^{\bowtie}$ email: nakanishi@chem.es.osaka-u.ac.jp
} 


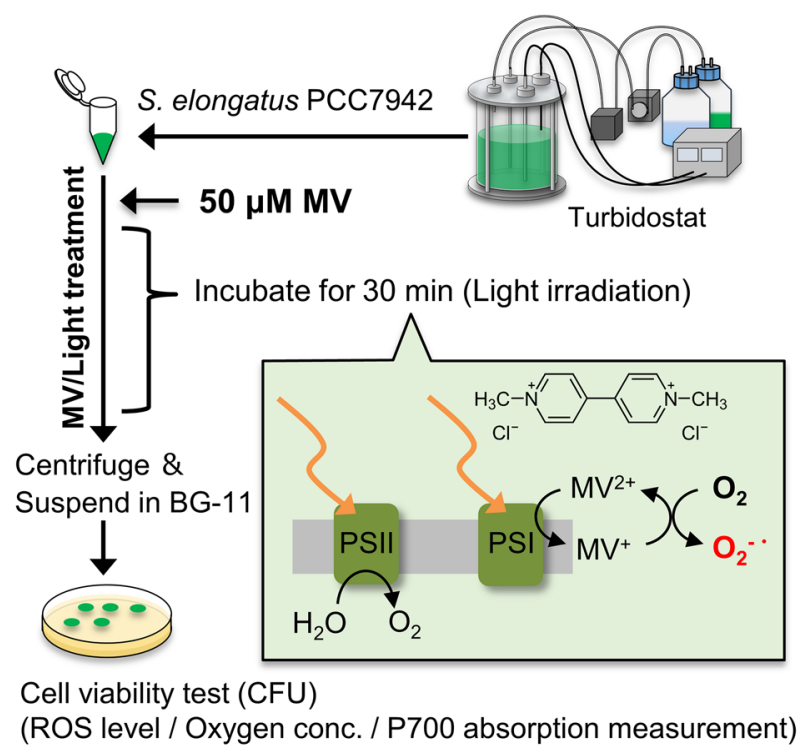

Figure 1. Schematic of $S$. elongatus growth, followed by ROS generation upon addition of MV with light incubation (MV/light treatment) and ROS tolerance evaluation. $M V$ methyl viologen, PSI photosystem I, PSII photosystem II.

Generally, in photosynthetic living cells, the generation of large amounts of ROS is triggered by unfavorable conditions such as strong light irradiation or exposure to high (or low) temperature. However, these environmental stimuli may mask the circadian control of oxidative stress tolerance because they directly affect a variety of metabolic pathways and induce adaptation mechanisms against oxidative stress ${ }^{10}$. Complexity of how ROS levels change in the harsh conditions might hinder the effect of circadian clock on oxidative stress tolerance. Therefore, we used methyl viologen (MV) with light irradiation as a specific ROS (i.e. superoxide, $\mathrm{H}_{2} \mathrm{O}_{2}$ and hydroxyl radical) generation system with the aim of evaluating circadian clock involvement in oxidative stress tolerance control at the phenotype level. Circadian rhythms of cell viability were observed when MV treatment with relatively low light intensity was used.

\section{Results}

ROS generation profile. The molecular mechanism of ROS generation by MV has been well clarified and is well suited for the present work. Specifically, under light irradiation, MV mediates an electron transfer reaction from photosystem I (PSI) or ferredoxin to oxygen, resulting in the production of superoxide ${ }^{11,12}$. The generated superoxide is converted to $\mathrm{H}_{2} \mathrm{O}_{2}$ and a hydroxyl radical by a subsequent disproportionation reaction and Fenton type reaction ${ }^{13}$. In our study, S. elongatus cells grown in a turbidostat were assayed using a protocol in which $50 \mu \mathrm{M}$ MV was added to cell culture aliquots, followed by $30 \mathrm{~min}$ light irradiation (this protocol for ROS generation is hereafter denoted "MV/light-treatment"). After MV/light-treatment, cells were washed with BG-11 medium and oxidative damage was evaluated by measuring oxygen evolution activity, P700 absorbance change or colony forming units (CFU) (Fig. 1).

Superoxide generated by the MV/light-treatment undergoes rapid disproportionation to hydrogen peroxide $\left(\mathrm{H}_{2} \mathrm{O}_{2}\right)$ and hydroxy radicals, which are ROS closely related to cellular viability. Therefore, we attempted to confirm the ROS generation by using $2^{\prime}, 7^{\prime}$-dichlorodihydrofluorescein diacetate $\left(\mathrm{H}_{2} \mathrm{DCFDA}\right)$, which is converted by intracellular esterases to $2^{\prime}, 7^{\prime}$-dichlorodihydrofluorescein, which can subsequently be oxidized by $\mathrm{H}_{2} \mathrm{O}_{2}$, hydroxyl and peroxyl radical to a fluorescent compound with an emission peak at around $520 \mathrm{~nm}$. To confirm light intensity dependency of ROS levels without the influence of the circadian clock, cyanobacterial cells were not entrained by LD ( $12 \mathrm{~h} \mathrm{light} / 12 \mathrm{~h}$ dark) cycles. The difference in fluorescence intensity was normalized to control samples incubated without MV in darkness, and showed a dependence on light intensity (Fig. 2A). A small increase in ROS levels was observed even for samples incubated in darkness with $50 \mu \mathrm{M} \mathrm{MV}$. We attribute this small increase to the reduction of MV by intracellular NADPH.

Although the assay using $\mathrm{H}_{2}$ DCFDA is an easy method for evaluating ROS level, samples containing $\mathrm{H}_{2}$ DCFDA cannot be cryopreserved due to their instability. Therefore, in order to confirm whether ROS level depends on the subjective time with $\mathrm{H}_{2} \mathrm{DCFDA}$, it is necessary to perform the sampling and measurement at once at each time point. To overcome this technical difficulty, electron paramagnetic resonance (EPR) method was adopted to measure the time variation of ROS level. With this technique, a sample containing the spin-trapping agent can be frozen and stored, allowing us to collect samples over time and then conduct high-throughput experiments. Specifically, in cell suspensions subjected to MV/light treatment at various subjective times, levels of ROS, including superoxide radicals, hydrogen peroxide, and hydroxyl radicals, were determined by EPR using 4-pyridyl-1-oxide- $N$-tert-butylnitrone as a spin-trapping agent. We observed circadian rhythms in ROS levels in both samples treated and non-treated by MV/light-treatment with light intensity of $10 \mu \mathrm{mol} \mathrm{m}^{-2} \mathrm{~s}^{-1}$ (Fig. 2B). 
A

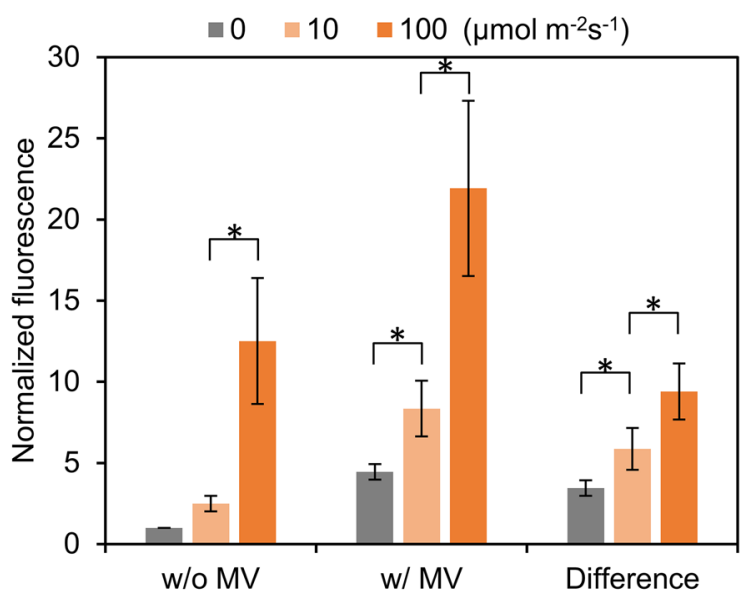

B

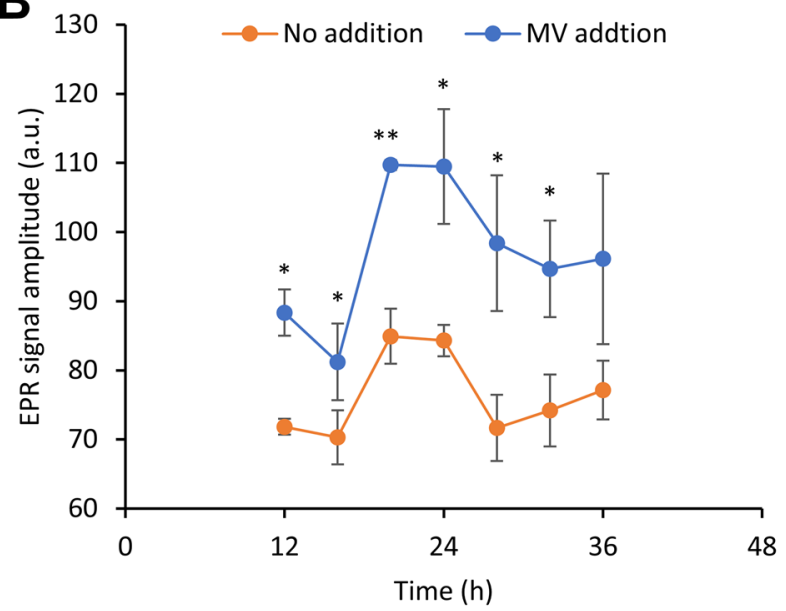

Figure 2. Effects of addition of MV, light irradiation intensity, and subjective time on intracellular ROS levels. (A) Wild type cells were incubated without MV (w/o MV) or with $50 \mu \mathrm{M} \mathrm{MV} \mathrm{(w/MV)} \mathrm{under} \mathrm{three} \mathrm{levels}$ of light intensity $\left(0,10\right.$, and $\left.100 \mu \mathrm{mol} \mathrm{m}^{-2} \mathrm{~s}^{-1}\right)$ for $30 \mathrm{~min}$. Fluorescence intensity of control samples (w/o $\mathrm{H}_{2}$ DCFDA) was subtracted from the fluorescence intensity of samples with $\mathrm{H}_{2}$ DCFDA, and the differences in fluorescence between with and without MV at each light intensity level are presented. Fluorescence intensity was measured from three biological replicates in each experiment. Values are means \pm SD (bars) results from three independent experiments (therefore, each bar is the average of $n=9)$. Student's $t$ tests were significant $\left({ }^{*} P<0.05\right)$. (B) Dependency of ROS levels in wild type cells on circadian time. Wild type cells sampled at various subjective times were treated with $10 \mu \mathrm{mol} \mathrm{m} \mathrm{m}^{-2} \mathrm{~s}^{-1}$ light irradiation for $30 \mathrm{~min}$, with or without $50 \mu \mathrm{M} \mathrm{MV}$. Generated hydroxyl radicals were determined by spin trapping EPR spectroscopy with 4-POBN. Values are means \pm SD (bars) of three biological replicates. Significant differences from samples without MV were evaluated by student's t tests $\left({ }^{*} P<0.05,{ }^{* *} P<0.001\right)$.

Although we cannot quantitatively determine absolute amount of ROS due to the presence of a certain level of baseline, the EPR signal clearly exhibited circadian rhythms. Furthermore, consistent with the results of the $\mathrm{H}_{2}$ DCFDA assay (Fig. 2A), MV/light treatment increased the average EPR signal value (Supplementary Fig. S1).

Effect of MV-induced oxidative stress on cell viability. Next, we examined the influence of MV/light treatment on cell viability. In the present paper, we defined the term "cell viability" was evaluated as the capacity of multiplying and forming colonies, which is one of the definitions of cell viability ${ }^{14}$. On this definition, the cell viability can be directly estimated through colony counting. Treated cells were cultured on agar plates, and the number of colonies after 6 or 7 days was counted (Fig. 3A). To eliminate the effect of the circadian clock, cells that were not entrained to the LD cycle were used. Although ROS levels increased when cells were incubated in darkness with MV (Fig. 2A), there were no significant differences in colony-forming units (CFU) between samples incubated in weak light irradiation $\left(10 \mu \mathrm{mol} \mathrm{m}^{-2} \mathrm{~s}^{-1}\right)$ without MV versus darkness with MV. In contrast, for MV/light treated cells, CFU values decreased in a light intensity-dependent manner, indicating that ROS gener- 
A

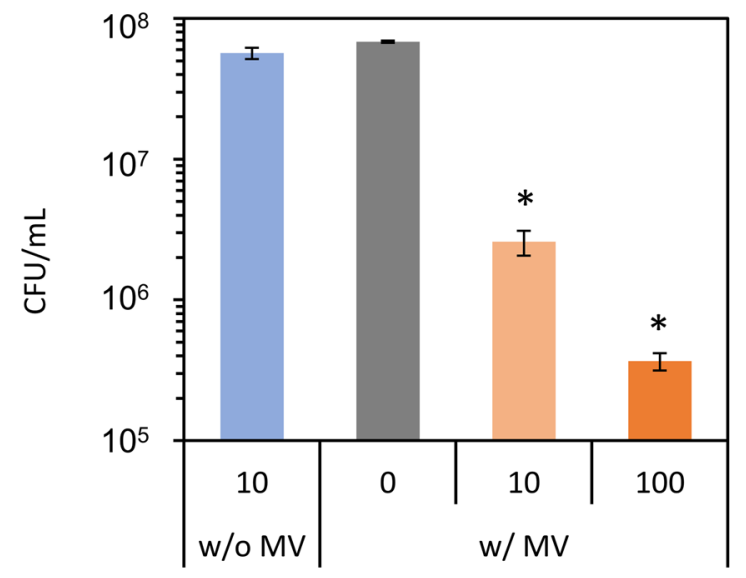

Light intensity $\left(\mu \mathrm{mol} \mathrm{m} \mathrm{m}^{-2} \mathrm{~s}^{-1}\right)$

C

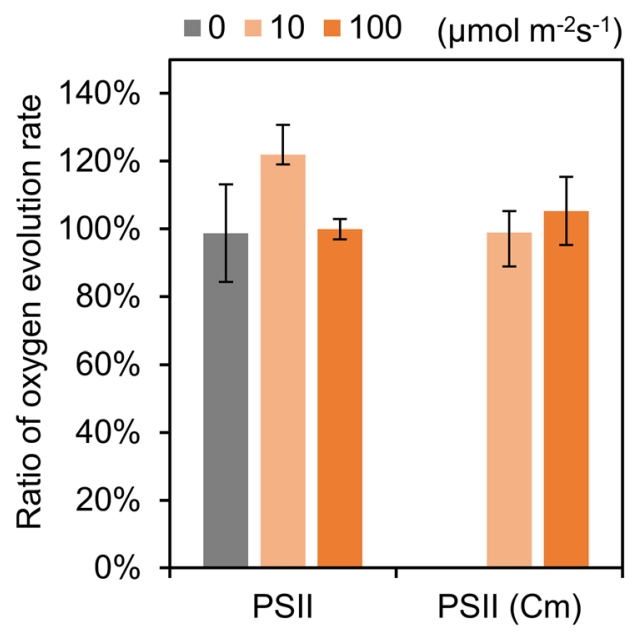

E

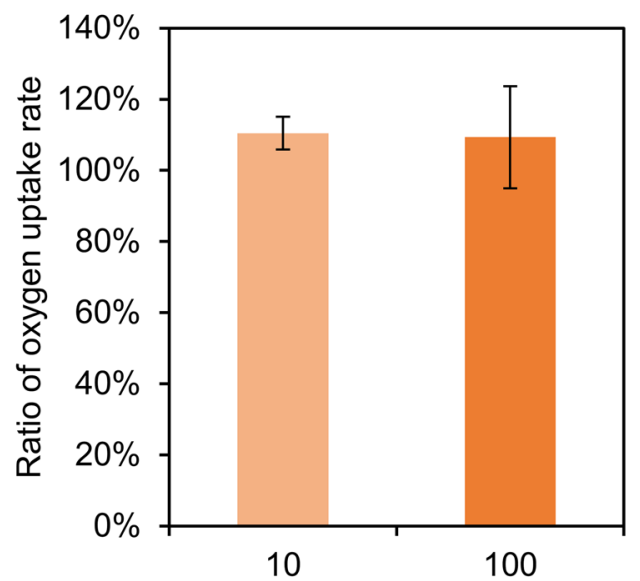

Light intensity $\left(\mu \mathrm{mol} \mathrm{m}^{-2} \mathrm{~s}^{-1}\right)$
B

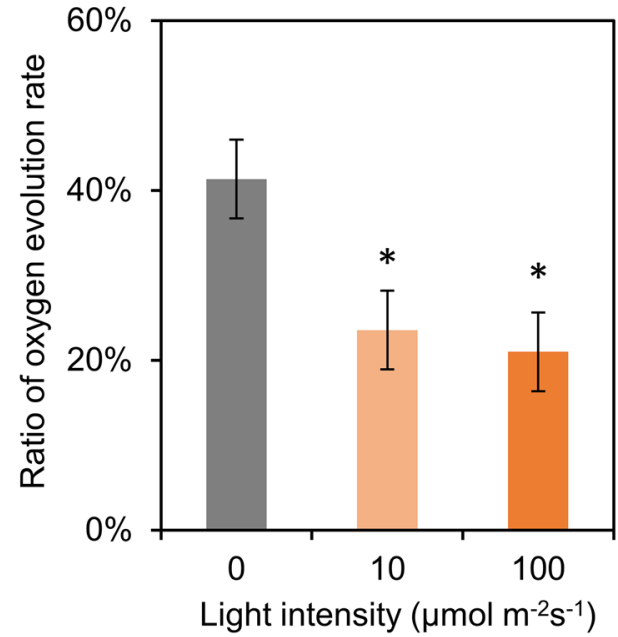

D

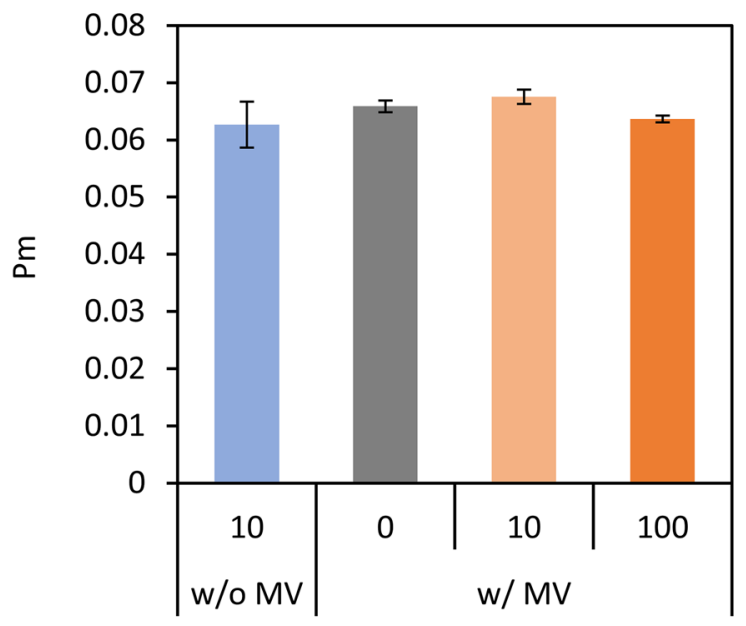

Light intensity $\left(\mu \mathrm{mol} \mathrm{m} \mathrm{s}^{-2}\right)$ 
४Figure 3. Evaluation of oxidative stress generated by MV/light treatment. (A) MV/light-treated cells were spotted on agar plates and grown to visualize the effects of MV/light treatment on cell viability. Obtained colonies were counted to calculate CFU. Values and error bars represent means \pm SD of three technical replicates. Significant differences from CFU without MV were evaluated by student's $t$ tests $\left({ }^{\star} P<0.01\right)$. (B,C) The ratio of the oxygen evolution rate of $10 \mu \mathrm{g} \mathrm{Chl} / \mathrm{mL}$ suspension-containing cells treated with and without $50 \mu \mathrm{M} \mathrm{MV}$ under three light intensities (MV/light treatment) was measured in the presence of (B) $\mathrm{NaHCO}_{3},(\mathbf{C}) \mathrm{DCBQ}$, or DCBQ and $200 \mu \mathrm{g} / \mathrm{mL}$ chloramphenicol, indicated as PSII and PSII (Cm), respectively. (D) Oxidizable P700 (Pm). (E) Ratio of oxygen uptake rate of $10 \mu \mathrm{g} \mathrm{Chl} / \mathrm{mL}$ suspension containing cells, treated with or without $50 \mu \mathrm{M}$ MV to examine MV/light treatment-induced oxidative damage of the photosynthetic electron transport chain, including components from PSII to PSI. For measurement of oxygen uptake, $1 \mathrm{mM} \mathrm{MV}, 1 \mathrm{mM} \mathrm{KCN}$ and $10 \mathrm{mM}$ methyl amine were added to a $10 \mu \mathrm{g} \mathrm{Chl} / \mathrm{mL}$ suspension containing MV/light-treated cells in an oxygen electrode chamber. (B-E) Values and error bars represent means \pm SD of three independent experiments. Significant differences from the samples treated in dark $\left(0 \mu \mathrm{mol} \mathrm{m} \mathrm{m}^{-2} \mathrm{~s}^{-1}\right)$ with $\mathrm{MV}$ were evaluated by student's $\mathrm{t}$ tests $\left({ }^{\star} P<0.05\right)$.

ated via the photosynthetic electron transport chain (PETC) through MV led to reduced cell viability. In addition, the finding that CFU changed in a light intensity-dependent manner during MV/light treatment means that CFU numbers were not influenced by residual MV during culture on agar plates, confirming that washing and serial dilution done prior to cell plating is sufficient to almost completely remove MV.

Effect of MV-induced oxidative stress on photosynthetic activity. To characterize oxidative stress damage by MV/light treatment in detail, we next investigated the effects of the treatment on the activities of overall electron transfer, including photosystem II (PSII) and photosystem I (PSI), activities. Overall electron transfer activity was evaluated by measuring the oxygen evolution reaction (OER) rate when $\mathrm{CO}_{2}\left(\mathrm{NaHCO}_{3}\right)$ was used as the sole electron acceptor. The ratio of the OER rate for cells incubated with MV versus that obtained for cells incubated without MV is shown in Fig. 3B and indicates that the OER rate markedly decreased following the addition of MV in the dark condition and decreased even more in light conditions. These patterns are consistent with normalized fluorescence data (Fig. 2A), which reveal light intensity-dependent increase in ROS levels following the MV treatment in both dark and light conditions.

Next, PSII activity was evaluated by measuring the OER rate in the presence of 2,6-dichlorobenzoquinone (DCBQ), which intercepts photosynthetic electrons, mainly from the $\mathrm{Q}_{\mathrm{B}}$ site in PSII. The OER rate was not affected by the addition of MV or light intensity (Fig. 3C) but was affected when $\mathrm{CO}_{2}$ was used as the electron acceptor (Fig. 3B). We propose that this is either because ROS generated by MV addition did not damage PSII or any damaged PSII was quickly repaired. To verify which of these two pathways led to the observed result, PSII activity measurements were next performed in the presence of a translation inhibitor, chloramphenicol $(\mathrm{Cm})$, which prevents repair of PSII ${ }^{15}$. Cells incubated in a medium containing $\mathrm{Cm}$ but not MV showed reduced PSII activity when exposed to light at an intensity of $100 \mu \mathrm{mol} \mathrm{m}^{-2} \mathrm{~s}^{-1}$ (Supplementary Fig. S2). However, PSII activity in the presence of $\mathrm{Cm}$ and at a light intensity of $10 \mu \mathrm{mol} \mathrm{m}^{-2} \mathrm{~s}^{-1}$ was maintained at the same level as that observed in the absence of $\mathrm{Cm}$. This indicates that photoinhibition of PSII does not occur under conditions of irradiation with $10 \mu \mathrm{mol} \mathrm{m} \mathrm{m}^{-2} \mathrm{~s}^{-1}$. In contrast, MV/light treatment did not affect PSII activity, irrespective of light intensity (Fig. 3C). These results indicate that ROS generated under MV/light treatment does not damage PSII.

Oxidative damage to PSI was also evaluated using an established protocol based on the absorption spectroscopy for the reaction center of PSI (chlorophyll, P700) ${ }^{16}$. As shown in Fig. 3D, the amount of oxidizable P700 $(\mathrm{Pm})$ in cells treated with MV was constant over the range of light intensity tested, indicating that MV/light treatment did not damage PSI.

Finally, the effect of MV-induced oxidative stress on PETC, including all components from PSII to PSI, was tested by measuring the light-induced $\mathrm{O}_{2}$ uptake rate, with $\mathrm{H}_{2} \mathrm{O}$ and $\mathrm{MV}$ serving as the electron donor and accepter, respectively. As shown in Fig. 3E, cells subjected to MV/light treatment maintained electron transfer activity at the same level for light irradiation at 10 and $100 \mu \mathrm{mol} \mathrm{m} \mathrm{m}^{-2}$. Taken together, generated ROS did not damage PETC, which is itself the machinery for generating ROS.

Oxidative damage to PSII or PSI is known to be caused by ROS generation due to excessive reduction of PETC components but not by ROS generation due to external addition of $\mathrm{H}_{2} \mathrm{O}_{2}$ or $\mathrm{MV}^{17-19}$. In good agreement with these studies, as mentioned earlier, PETC activity was not impacted by oxidative stress induced by MV/ light treatment. Nevertheless, overall photosynthetic activity and CFU values were decreased following MV/light treatment (Fig. 3A,B). These results clearly indicate that stromal components, including enzymes participating in electron flux downstream of PSI, were damaged by ROS. In fact, lipids, DNA and Rubisco, which has $\mathrm{CO}_{2}$ fixing ability, are known to be damaged by ROS generated by $\mathrm{MV}^{20,21}$. Oxidative damage to such components is likely to have led to a decrease in overall electron transfer activity and CFU.

Effect of the circadian clock on MV-induced oxidative stress tolerance. To investigate circadian control of oxidative stress tolerance, MV/light treatment was used to generate ROS in cells sampled at various subjective times. For the circadian tests, cells cultured in a turbidostat were kept at a constant cell density (optical density $730 \mathrm{~nm}\left(\mathrm{OD}_{730}\right)=0.6 \pm 0.1$, Supplementary Fig. S3) in order to exclude the possibility that differences in growth stage influence the CFU results. After subjecting the cells to LD cycles with $24 \mathrm{~h}$ periodicity, cells were exposed to continuous light (LL) conditions in the turbidostat.

First of all, the MV/light treatment was processed under the light intensity of growth condition $\left(40 \mu \mathrm{mol} \mathrm{m} \mathrm{m}^{-2} \mathrm{~s}^{-1}\right)$, since MV treatment is generally performed at the light intensity of standard growth 
A

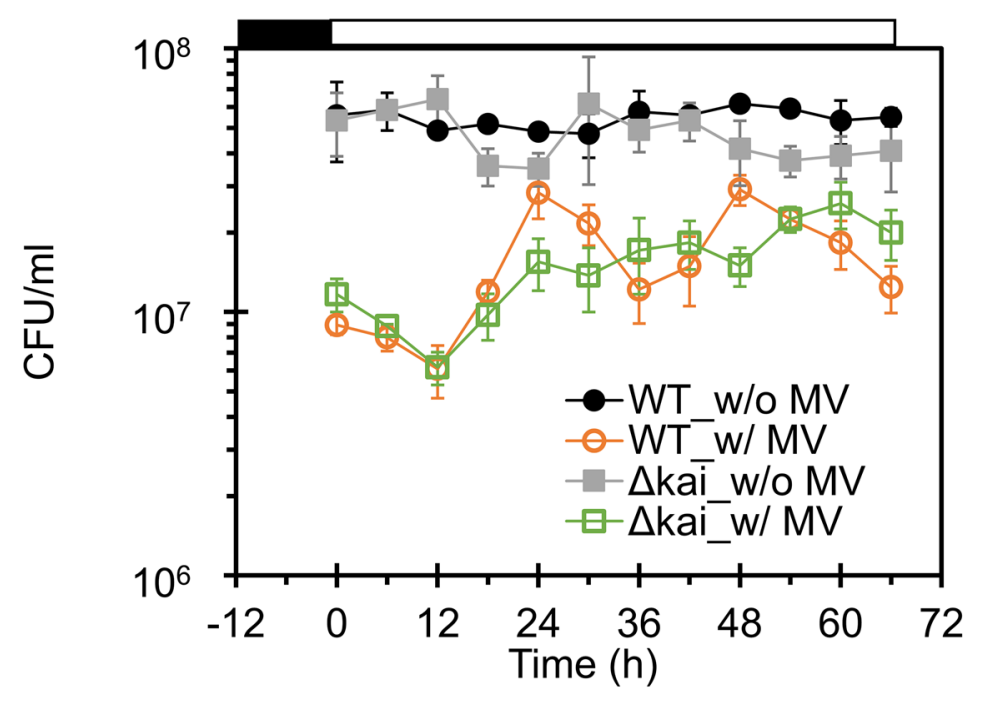

B

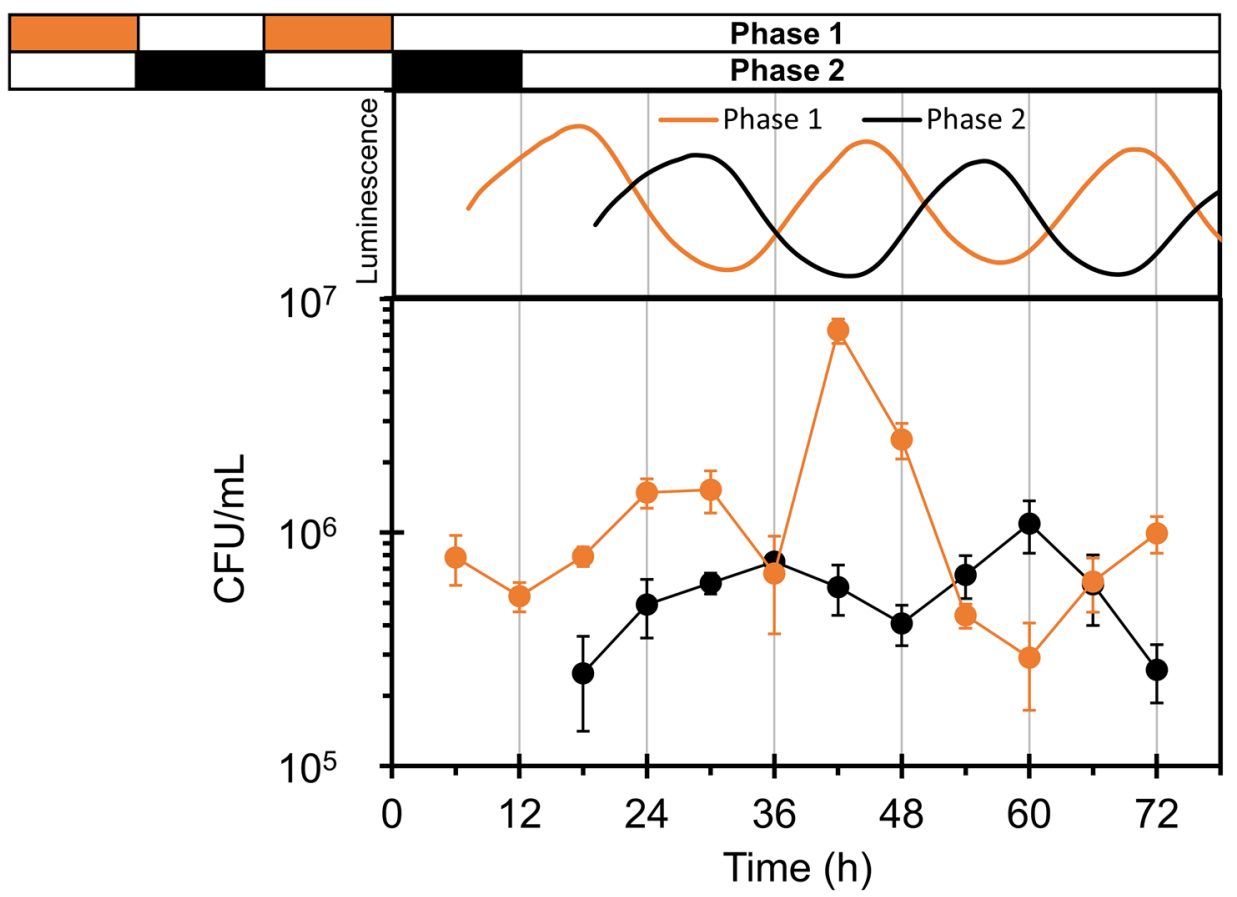

Figure 4. Time series of CFU of MV/light treated cells. (A) Wild type (WT) and kaiABC-deficient mutant $(\triangle \mathrm{kai} A B C)$ cells were harvested from turbidostat every $6 \mathrm{~h}$, followed by MV/light treatment $\left(10 \mu \mathrm{mol} \mathrm{m}^{-2} \mathrm{~s}^{-1}\right)$ of each aliquot. Oxidative stress tolerance was visualized as the number of colonies. (B) ROS tolerance of WT cell cultures entrained in antiphase were tested every $6 \mathrm{~h}$. Luminescence rhythms of WT cells were also measured to identify the phase of each culture, and are shown above the CFU graph. White and black or orange bars denote light-dark (LD) cycles. Values and error bars represent means \pm SD of three technical replicates.

conditions $^{22-24}$. However, in this case, the effect of circadian clock on cell viability (i.e., CFU) was not clearly detected (Supplementary Fig. S4). We assumed that large amount of ROS generated in this condition directly stimulated the physiological responses of the cells and masked the physiology controlled by circadian clock, and then reduced the light intensity. When $10 \mu \mathrm{mol} \mathrm{m} \mathrm{m}^{-2} \mathrm{~s}^{-1}$ was applied for MV/light treatment, catalase and SOD activity were stable during the treatment, indicating that MV/light treatment with lower light intensity induce relatively mild oxidative stress and hardly induce subsequent adaptation responses, as expected (Supplementary Fig. S5).

On this condition, as shown in Fig. 4A and Supplementary Figs. S6 S8, while non-treated wild type (WT) cells (filled circles) showed no dependence on subjective time, the MV/light-treated cells showed circadian rhythm (open circles). Mutant cells lacking clock genes $(\triangle k a i A B C)$ did not show clear rhythms, whether or not they were treated with MV/light (closed and open squares in Fig. 4A). It should be noted that the circadian 
A
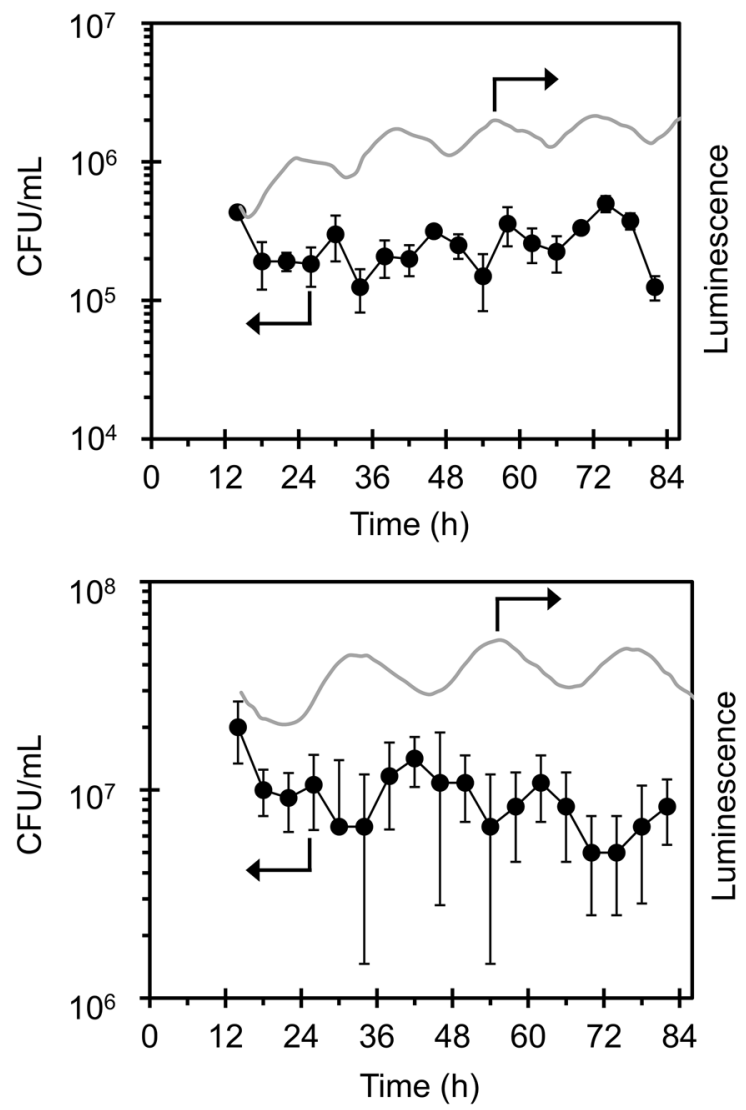

Figure 5. Correlation of period length between gene expression rhythms and ROS tolerance rhythms of period mutant strains. ROS tolerance of (A) F470Y and (B) S157P strains cultured in continuous light conditions following $12 \mathrm{~h}$ light/ $12 \mathrm{~h}$ dark cycles for entrainment were tested. Gray lines indicate luminescence traces of the cells, which confirm the expected short-period phenotype. Values represent means of three technical replicates.

rhythmicity was evaluated after $12 \mathrm{~h}$, following the general protocol for the studies on circadian rhythms. This is because of that the condition in the first $12 \mathrm{~h}$ period after switching to continuous light condition is exactly the same as that under the LD cycle. Importantly, the oscillatory phase of the CFU rhythm was determined by the phase of LD cycles (Fig. 4B). Further, using period mutant cells (F470Y, S157P) with a free running period (FRP) of 16 and $21 \mathrm{~h}$, respectively ${ }^{25}$, CFU results showed a periodicity that corresponded with the FRP (Fig. 5).

\section{Discussion}

As shown above, the circadian rhythms of oxidative stress tolerance were experimentally observed by using $\mathrm{MV} /$ light treatment, which causes oxidative damage in stromal components (Figs. 2, 3, 4). In the kaiABC deletion mutant, the CFU rhythm disappeared (Fig. 4A). In addition, short-period mutants showed CFU rhythms corresponding to each FRP (Fig. 5). These results clearly indicate that ROS management of the KaiABC system affects cell viability in a time-of-day-dependent manner.

Light intensity during MV/light treatment is an important parameter because it directly affected ROS level and CFU (Figs. 2, 3). When the MV/light treatment was processed under the light intensity of the growth condition $\left(40 \mu \mathrm{mol} \mathrm{m}{ }^{-2} \mathrm{~s}^{-1}\right)$, the circadian rhythm of CFU values became unclear (Supplementary Fig. S4). On the other hand, the CFU values exhibit a circadian rhythm under the "mild oxidative stress" conditions at MV/light treatment with a light intensity of $10 \mu \mathrm{mol} \mathrm{m} \mathrm{s}^{-2}$, where catalytic activities of catalase and SOD were not affected (Supplementary Fig. S5) but the CFU value was significantly reduced (Fig. 3). These results indicate that the rhythm of CFU values (i.e., cell viability) is a phenomenon that occurs under "mild oxidative stress" conditions. While the expression of ROS-scavenging enzymes, such as peroxiredoxin and SOD, is a (post-)response to ROS generation $^{10}$, the circadian clock is the (pre-)adaptation system against the periodic change in the environmental light. This difference in the role might be the reason why the light intensity region for the appearance of the circadian rhythm in ROS-stress tolerance is smaller than that for the (post-)responsive mechanisms.

The time-of-day-dependent change in cell viability can arise from rhythmic variation in ROS levels (case-I) and/or by rhythmic damage of a critical component for cell viability (case-II), the latter of which can occur even at a constant ROS level. Considering that the circadian clock based on the KaiABC system is a global regulator, the effects of both of these factors are likely to change in a circadian manner. 
First, let us consider case-I. As shown Fig. 2B, the ROS level of samples in natural conditions (i.e. without $\mathrm{MV} /$ light treatment) also varied in a subjective time-of-day-dependent manner. ROS levels are defined by the balance between the amount of ROS generated and the amount scavenged. In general, accumulation of ROS occurs when electron acceptor capacity at the PSI side is not high enough as compared with electron flux from the donor side ${ }^{18}$. Independent transcriptome studies showed that the expression patterns of genes encoding photosynthetic components related to the electron acceptor capacity at PSI, such as genes encoding Rubisco and GAPDH, are rhythmic under the control of KaiABC ${ }^{7,26}$. Thus, it is highly likely that the amount of ROS generated oscillates in a circadian manner, even in LL conditions. On the other hand, generated superoxide and $\mathrm{H}_{2} \mathrm{O}_{2}$ should be scavenged by antioxidant enzymes such as superoxide dismutase (SOD) and catalase. According to the microarray analyses, genes encoding of 2-cys peroxiredoxin, SOD and catalase are rhythmically expressed even in a LL condition ${ }^{7,26}$. These facts suggest that circadian variation in ROS levels is due at least in part to rhythmic gene expression as controlled by KaiABC.

Importantly, although circadian fluctuations in ROS levels were observed in both the presence and absence of MV/light treatment (Fig. 2B), circadian fluctuations in CFU were observed only for MV/light-treated cells (Fig. 4A). This inconsistence led us to hypothesize that cell viability changes only when ROS levels exceed a certain threshold. In other words, without MV/light treatment, the amount of ROS is not high enough to cause a decrease in CFU even at peak levels and thus, time-of-day-dependent changes in CFU were not observed. When the cells were MV/light-treated, average ROS levels were increased at all time points (Fig. 2B). However, ROS levels exceeded the threshold in subjective midnight, leading to circadian variation in cell viability. Consistent with this hypothesis, despite the fact that the addition of MV in dark increased ROS levels (Fig. 2A) and decreased photosynthetic activity (Fig. 3B), CFU values in this case showed no differences from those obtained in the absence of MV (Fig. 3A). The phases of circadian rhythms of ROS levels and CFU variation did not coincide: ROS levels peaked during a period from subjective midnight to dawn, whereas CFU peaked around subjective dawn. This could be because the ROS-induced response continues for several hours after MV/light treatment.

We also considered the possibility that rhythmic damage to a critical component required for cell viability (i.e., case-II) is the origin of the time-of-day-dependent variation. For example, it is known that cell division is controlled by circadian clock in order to help cope with oxidative stress ${ }^{3}$. Furthermore, in S. elongatus, the circadian clock regulates gating of cell division ${ }^{27}$. Considering these reports, we reason that circadian rhythm in CFU might have appeared due to the influence of circadian gating of cell division. We speculate that both of these two cases work in parallel and under the regulation of KaiABC, together leading to time-of-day-dependent changes in CFU that we observed.

In summary, this study experimentally demonstrated for the first time that ROS levels and cell viability in response to oxidative stress change in a circadian manner under the control of KaiABC. Circadian variation in CFU in response to MV/light treatment was observed in specific conditions (MV concentration: $50 \mu \mathrm{M}$, light intensity: $10 \mu \mathrm{mol} \mathrm{m} \mathrm{m}^{-2} \mathrm{~s}^{-1}$, and light irradiation: $30 \mathrm{~min}$ ). In principle, rhythmicity in CFU might also be observed, for example, at higher light intensity if the MV concentration was reduced or no MV was added. An important finding obtained throughout this work is that the intracellular ROS levels (case-I) and/or ROS sensitivity of a specific cellular component (case-II) can show circadian oscillation. We anticipate that further studies along this line will contribute to understanding competitive fitness advantages, which have attracted attention from the viewpoint of understanding the origin and evolution of the circadian clock $^{1,4}$.

\section{Methods}

Bacterial strains and cell culture conditions. We used the following $S$. elongatus strains: wild-type reporter strains $\left(\mathrm{P}_{k a i B C}:: l u x A B \text { and } \mathrm{P}_{p s b A r}: \text { lux } C D E\right)^{28}$, a kaiABC-deficient $\mathrm{P}_{k a i B C}$ reporter strain ${ }^{29}$ and two kaiC mutant strains with short periodicity carrying $\mathrm{P}_{k a i B C}$ reporters (F470Y and $\left.\mathrm{S} 157 \mathrm{P}\right)^{25}$. These strains were grown in liquid or on solid (1.5\% Bacto agar) BG-11 medium. Liquid cultures were grown in flasks (batch culture) or turbidostat (continuous culture) at $30{ }^{\circ} \mathrm{C}$ with air bubbling and exposure to light at an intensity of $30-40 \mu \mathrm{mol} \mathrm{m}^{-2} \mathrm{~s}^{-1}$. For continuous culture, cell density was continuously monitored using an optical sensor with near-infrared light (NI; $840 \mathrm{~nm} \sim 910 \mathrm{~nm}$ ) and cell density was controlled by dilution with fresh medium when the OD value in the $\mathrm{NI}$ region exceeded a certain value to maintain $\mathrm{OD}_{730}$ is $0.65 \pm 0.05$.

Methyl viologen (MV) treatment. $\quad \mathrm{MV}$ was added at a final concentration of $50 \mu \mathrm{M}$ to liquid culture medium to achieve an optical density at $730 \mathrm{~nm}\left(\mathrm{OD}_{730}\right)$ of $0.65 \pm 0.05$. Negative control samples without $\mathrm{MV}$ were prepared at the same time. The samples were incubated for $30 \mathrm{~min}$ at the specified light intensity, and cells were collected by 5 -min centrifugation at $4400 \times g$ at $30^{\circ} \mathrm{C}$, re-suspended in fresh BG-11 medium to remove MV, centrifuged again, and re-suspended again in fresh BG-11 to produce the cell density required for subsequent measurements.

$\mathrm{H}_{2}$ DCFDA assay. Intracellular ROS levels were measured by using $\mathrm{H}_{2}$ DCFDA as described previously with slight modifications ${ }^{6,30}$. Just before the assay, $\mathrm{H}_{2}$ DCFDA (Invitrogen) was dissolved in ethanol to produce a $15 \mathrm{mM}$ stock solution. The $\mathrm{H}_{2} \mathrm{DCFDA}$ stock solution or ethanol (vehicle) was then added to a liquid culture of $\mathrm{OD}_{730}$ of $0.65 \pm 0.05$ and the treated culture was incubated in darkness for $30 \mathrm{~min}$ at $30^{\circ} \mathrm{C}$. Next, $\mathrm{H}_{2} \mathrm{DCFDA}$ was removed from the culture medium by centrifugation, the culture medium was combined with an equal amount of fresh BG-11 medium, and $50 \mu \mathrm{M}$ MV was or was not added. To generate ROS, the cell suspension was incubated for $30 \mathrm{~min}$ at the indicated light intensity at $30^{\circ} \mathrm{C}$. After light treatment, the cells were placed in a 96 -well plate and the fluorescence emission at $535 \mathrm{~nm}$ was measured ( $485 \mathrm{~nm}$ excitation) on a Tecan Infinite M200 Plate Reader. The background fluorescence signal of samples without $\mathrm{H}_{2}$ DCFDA (ethanol only) was subtracted from the fluorescence values of the corresponding samples with $\mathrm{H}_{2}$ DCFDA. 
Spin trapping EPR spectroscopy. Spin trapping assays with the spin probe 4-pyridyl-1-oxide- $N$-tertbutylnitrone (4-POBN; Sigma-Aldrich, St. Louis, USA) to detect the formation of hydroxyl radicals ${ }^{31}$ were carried out using cyanobacterial cells $\left(\mathrm{OD}_{730}=0.65\right)$ in $10 \mathrm{mM}$ sodium phosphate buffer $(\mathrm{pH} 7.0)$ containing $50 \mathrm{mM}$ 4-POBN, 4\% (v/v) ethanol, $50 \mu \mathrm{M}$ Fe-EDTA, and $50 \mu \mathrm{M} \mathrm{MV}$. After a 30-min incubation in the light $\left(10 \mu \mathrm{mol} \mathrm{m}{ }^{-2} \mathrm{~s}^{-1}\right)$, the samples were centrifuged at $7000 \mathrm{~g}$ for $1 \mathrm{~min}$, and the supernatants were frozen in liquid nitrogen and stored at $-80^{\circ} \mathrm{C}$ for electron paramagnetic resonance (EPR) spectra analysis. The EPR spectra were recorded at room temperature in a standard quartz flat cell using an ESP-300 X-band spectrometer (Bruker, Rheinstetten, Germany). The following parameters were used: microwave frequency, $9.73 \mathrm{GHz}$; modulation frequency, $100 \mathrm{kHz}$; modulation amplitude, $1 \mathrm{G}$; microwave power, 6.3 milliwatt; receiver gain, $2 \times 104$; time constant, $40.96 \mathrm{~ms}$; number of scans: 4 .

Measurement of oxygen concentration. Reaction mixtures containing BG-11 with 40 mM TES-NaOH ( $\mathrm{pH}$ 7.5), $50 \mathrm{mM} \mathrm{NaHCO}$ and cells after MV/light treatment $(10 \mu \mathrm{g} \mathrm{Chl} / \mathrm{mL})$ were stirred with a magnetic stirrer, and the oxygen concentration was monitored using an oxygen electrode (Hansatech, UK). Overall activity was determined by light irradiation with an LED source (pE-100 wht, BioVision Technologies, USA) through a $550 \mathrm{~nm}$ long pass filter at light intensity of $660 \mu \mathrm{mol} \mathrm{m}^{-2} \mathrm{~s}^{-1}$. Next, $0.4 \mathrm{mM}$ 2,6-dichlorobenzoquinone (DCBQ) was added to determine PSII activity. To determine electron transfer activity from PSII to PSI, light-induced oxygen uptake of MV/light-treated cells was measured in the presence of $1 \mathrm{mM} \mathrm{KCN,} 1 \mathrm{mM} \mathrm{MV}$, and $10 \mathrm{mM}$ methyl amine.

Photochemical measurement of total P700. Absorbance changes of P700 (reaction center chlorophyll of PSI) were monitored using a fiber version of the Dual-PAM-100 measuring system at room temperature. Samples containing BG-11 with $40 \mathrm{mM}$ TES-NaOH (pH 7.5) and cells post-MV/light treatment (15 $\mu \mathrm{g}$ $\mathrm{Chl} / \mathrm{mL}$ ) were introduced into a cuvette. To measure maximum P700 absorption, saturating pulse illumination $\left(10,000 \mu \mathrm{mol} \mathrm{m}^{-2} \mathrm{~s}^{-1}\right)$ was applied after irradiation with blue light $\left(750 \mu \mathrm{mol} \mathrm{m}{ }^{-2} \mathrm{~s}^{-1}\right)$ for $5 \mathrm{~s}$.

Viable cell plating. Samples after MV/light treatment were re-suspended in fresh BG-11 to reach an $\mathrm{OD}_{730}$ of 0.3. Each sample was serially diluted 1:10 four times in fresh BG-11 and then $4 \mu \mathrm{L}$ of each diluted sample was spotted onto solid BG-11 plates without antibiotics. The plates were incubated at $30{ }^{\circ} \mathrm{C}$ under $30 \mu \mathrm{mol} \mathrm{m}^{-2} \mathrm{~s}^{-1}$ constant light for 6 or 7 days and then photographed.

Statistical analyses. Statistical analyses (Student's t-test and ANOVA) were performed in Microsoft excel. To assess the rhythmicity of CFU time series of WT and $\triangle k a i A B C$ strain, the CFU data were de-trended linearly, which is generally performed for rhythm analyses ${ }^{32,33}$. Significance of the time-dependency of the de-trended data were analyzed by one-way ANOVA.

\section{Data availability}

Data supporting the findings of this paper are available from the corresponding authors upon reasonable request.

Received: 27 July 2020; Accepted: 3 November 2020

Published online: 18 November 2020

\section{References}

1. Edgar, R. S. et al. Peroxiredoxins are conserved markers of circadian rhythms. Nature 485, 459-464 (2012).

2. Lai, A. G. et al. CIRCADIAN CLOCK-ASSOCIATED 1 regulates ROS homeostasis and oxidative stress responses. Proc. Natl. Acad. Sci. 109, 17129-17134 (2012).

3. Miyagishima, S. et al. Translation-independent circadian control of the cell cycle in a unicellular photosynthetic eukaryote. Nat. Commun. 5, 3807 (2014).

4. Johnson, C. H., Zhao, C., Xu, Y. \& Mori, T. Timing the day: What makes bacterial clocks tick?. Nat. Rev. Microbiol. 15, 232-242 (2017).

5. Swan, J. A., Golden, S. S., LiWang, A. \& Partch, C. L. Structure, function, and mechanism of the core circadian clock in cyanobacteria. J. Biol. Chem. 293, 5026-5034 (2018).

6. Diamond, S. et al. Redox crisis underlies conditional light-dark lethality in cyanobacterial mutants that lack the circadian regulator, RpaA. Proc. Natl. Acad. Sci. 114, E580-E589 (2017).

7. Ito, H. et al. Cyanobacterial daily life with Kai-based circadian and diurnal genome-wide transcriptional control in Synechococcus elongatus. Proc. Natl. Acad. Sci. 106, 14168-14173 (2009).

8. Guerreiro, A. C. et al. Daily rhythms in the cyanobacterium Synechococcus elongatus probed by high-resolution mass spectrometrybased proteomics reveals a small defined set of cyclic proteins. Mol. Cell. Proteom. 13, 2042-2055 (2014).

9. Cervený, J., Sinetova, M. A., Valledor, L., Sherman, L. A. \& Nedbal, L. Ultradian metabolic rhythm in the diazotrophic cyanobacterium Cyanothece sp. ATCC 51142. Proc. Natl. Acad. Sci. 110, 13210-13215 (2013).

10. Latifi, A., Ruiz, M. \& Zhang, C. Oxidative stress in cyanobacteria. FEMS Microbiol. Rev. 33, 258-278 (2009).

11. Farrington, J. A., Ebert, M., Land, E. J. \& Fletcher, K. B. Bipyridylium quaternary salts and related compounds. V. Pulse radiolysis studies of the reaction of paraquat radical with oxygen. Implications for the mode of action of bipyridyl herbicides. Biochim. Biophys. Acta 314, 372-381 (1973).

12. Sétif, P. Electron-transfer kinetics in cyanobacterial cells: Methyl viologen is a poor inhibitor of linear electron flow. Biochim. Biophys. Acta 1847, 212-222 (2015).

13. Babbs, C. F., Pham, J. A. \& Coolbaugh, R. C. Lethal hydroxyl radical production in paraquat-treated plants. Plant Physiol. 90, 1267-1270 (1989).

14. Roszak, D. B. \& Colwell, R. R. Survival strategies of bacteria in the natural environment. Microbiol. Rev. 51, 365-379 (1987).

15. Nishiyama, Y., Allakhverdiev, S. I., Yamamoto, H., Hayashi, H. \& Murata, N. Singlet oxygen inhibits the repair of photosystem II by suppressing the translation elongation of the D1 Protein in Synechocystis sp. PCC 6803. Biochemistry 43, 11321-11330 (2004). 
16. Christof, K. \& Ulrich, S. An improved method, using saturating light pulses, for the determination of photosystem I quantum yield via P700 -absorbance changes at $830 \mathrm{~nm}$. Planta 192, 261-268 (1994).

17. Herbert, S. K., Samson, G., Fork, D. C. \& Laudenbach, D. E. Characterization of damage to photosystems I and II in a cyanobacterium lacking detectable iron superoxide dismutase activity. Proc. Natl. Acad. Sci. 89, 8716-8720 (1992).

18. Sonoike, K. Degradation of $p s a B$ gene product, the reaction center subunit of photosystem I, is caused during photoinhibition of photosystem I: Possible involvement of active oxygen species. Plant Sci. 115, 157-164 (1996).

19. Allakhverdiev, S. I. \& Murata, N. Environmental stress inhibits the synthesis de novo of proteins involved in the photodamagerepair cycle of Photosystem II in Synechocystis sp. PCC 6803. Biochim. Biophys. Acta 1657, 23-32 (2004)

20. Desimone, M., Henke, A. \& Wagner, E. Oxidative stress induces partial degradation of the large subunit of ribulose-1,5-bisphosphate carboxylase/oxygenase in isolated chloroplasts of barley. Plant Physiol. 111, 789-796 (1996).

21. Imlay, J. A. Pathways of oxidative damage. Annu. Rev. Microbiol. 57, 395-418 (2003).

22. Hishiya, S. et al. Binary reducing equivalent pathways using NADPH-thioredoxin reductase and ferredoxin-thioredoxin reductase in the cyanobacterium Synechocystis sp. strain PCC 6803. Plant Cell Physiol. 49, 11-18 (2008).

23. Mihara, S., Yoshida, K., Higo, A. \& Hisabori, T. Functional significance of NADPH-thioredoxin reductase C in the antioxidant defense system of cyanobacterium Anabaena sp. PCC 7120. Plant Cell Physiol. 58, 86-94 (2017).

24. Hakkila, K., Valev, D., Antal, T., Tyystjarvi, E. \& Tyystjarvi, T. Group 2 sigma factors are central regulators of oxidative stress acclimation in cyanobacteria. Plant Cell Physiol. 60, 436-447 (2019).

25. Nakajima, M. et al. Reconstitution of circadian oscillation of cyanobacterial KaiC phosphorylation in vitro. Science 308, 414-415 (2005).

26. Vijayan, V., Zuzow, R. \& O’Shea, E. K. Oscillations in supercoiling drive circadian gene expression in cyanobacteria. Proc. Natl. Acad. Sci. 106, 22564-22568 (2009).

27. Mori, T., Binder, B. \& Johnson, C. H. Circadian gating of cell division in cyanobacteria growing with average doubling times of less than 24 hours. Proc. Natl. Acad. Sci. 93, 10183-10188 (1996).

28. Lu, Y. et al. Regulation of the cyanobacterial circadian clock by electrochemically controlled extracellular electron transfer. Angew. Chem. Int. Ed. Engl. 53, 2208-2211 (2014).

29. Ishiura, M. et al. Expression of a gene cluster kaiABC as a circadian feedback process in cyanobacteria. Science 281, 1519-1524 (1998).

30. Sae-Tang, P. et al. Overexpressed superoxide dismutase and catalase act synergistically to protect the repair of PSII during photoinhibition in Synechococcus elongatus PCC 7942. Plant Cell Physiol. 57, 1899-1907 (2016).

31. Michelet, L. \& Krieger-Liszkay, A. Reactive oxygen intermediates produced by photosynthetic electron transport are enhanced in short-day grown plants. Biochim. Biophys. Acta Bioenergy. 1817, 1306-1313 (2012).

32. Kucho, K. et al. Global analysis of circadian expression in the cyanobacterium Synechocystis sp. strain PCC 6803. J. Bacteriol. 187, 2190-2199 (2005).

33. Ma, P., Mori, T., Zhao, C., Thiel, T. \& Johnson, C. H. Evolution of KaiC-dependent timekeepers: A proto-circadian timing mechanism confers adaptive fitness in the purple bacterium Rhodopseudomonas palustris. PLoS Genet. 12, e1005922 (2016).

\section{Acknowledgements}

We thank Prof. K. Terauchi (Ritsumeikan University) and Prof. T. Kondo (Nagoya University) for kindly providing the mutant strains used in this work; Prof. K. Tanaka and Mr. T. Tsurumaki (Tokyo Institute of Technology) for providing a key suggestion regarding clock-controlled ROS tolerance through a presentation at the 59th Annual Meeting of the Japanese Society of Plant Physiologists (Sapporo, Japan 2018); Prof. A. Krieger-Liszkay for critical reading of the manuscript and providing valuable comments. This work was partially supported by the Advanced Low Carbon Technology Research and Development Program (JPMJAL1402) of the Japan Science and Technology Agency (JST), JSPS KAKENHI (19K22232 and 18J20176), and a JSPS oversea research fellowship (201860126).

\section{Author contributions}

K.T. and S.N. designed the research. G.S. determined ROS levels by spin trapping EPR spectroscopy. K.T. performed all of the other experiments. K.T. designed the experiments. K.T. and S.N. wrote the first draft of the manuscript. K.T. prepared figures. All authors (K.T., G.S. and S.N.) edited the manuscript.

\section{Competing interests}

The authors declare no competing interests.

\section{Additional information}

Supplementary information is available for this paper at https://doi.org/10.1038/s41598-020-77141-8.

Correspondence and requests for materials should be addressed to S.N.

Reprints and permissions information is available at www.nature.com/reprints.

Publisher's note Springer Nature remains neutral with regard to jurisdictional claims in published maps and institutional affiliations.

Open Access This article is licensed under a Creative Commons Attribution 4.0 International License, which permits use, sharing, adaptation, distribution and reproduction in any medium or format, as long as you give appropriate credit to the original author(s) and the source, provide a link to the Creative Commons licence, and indicate if changes were made. The images or other third party material in this article are included in the article's Creative Commons licence, unless indicated otherwise in a credit line to the material. If material is not included in the article's Creative Commons licence and your intended use is not permitted by statutory regulation or exceeds the permitted use, you will need to obtain permission directly from the copyright holder. To view a copy of this licence, visit http://creativecommons.org/licenses/by/4.0/.

(C) The Author(s) 2020 